\title{
IEEE 802.21-enabled ALIVE-HO for Media Streaming in Heterogeneous Wireless Networks
}

\author{
Liping Chen \\ Department of Computer Science \\ Trinity College Dublin, Ireland \\ Email: lipingc@scss.tcd.ie
}

\author{
Ahmed H. Zahran \\ Mobile \& Internet Systems Laboratory \\ Department of Computer Science \\ University College Cork, Ireland \\ Email:a.zahran@cs.ucc.ie
}

\author{
Cormac J. Sreenan \\ Mobile \& Internet Systems Laboratory \\ Department of Computer Science \\ University College Cork, Ireland \\ Email: cjs@cs.ucc.ie
}

\begin{abstract}
The convergence of heterogeneous wireless access technologies is an intrinsic part of the long term evolution of wireless networks. This convergence creates network overlays in which achieving seamless and efficient roaming between different technologies, commonly known as vertical handoff (VHO), introduces several challenging design issues. The media independent handover standard (IEEE 802.21) enables information exchange across different layers to improve VHO performance. In this work, we present a framework for integrating an adaptive lifetime-based vertical handoff (ALIVE-HO) algorithm with IEEE 802.21. The proposed framework is implemented and the optimal design value of the application parameter of ALIVE-HO is experimentally determined for video streaming applications under different operating scenarios.
\end{abstract}

\section{INTRODUCTION}

Next-generation wireless networks will integrate different access technologies, such as cellular, WiMAX, WiFi, to offer multimedia services to end users [1]. These wireless technologies have complementary characteristics in terms of cost, coverage, and resources. Hence, the service convergence of these technologies will help the operators improve the utilization of their scarce wireless resources and will enable the user to be always connected to the best available wireless network in the vicinity.

In integrated systems, vertical handoff (VHO) represents a unique handoff process due to the inherent system heterogeneity of different access technologies. In traditional horizontal handoff (HHO), the decision is mainly based on comparing the received signal strength (RSS) from candidate point of attachments (PoA) [2]. In VHO, the decision parameters, including the RSSs, are incomparable due to the heterogeneity of candidate PoAs. Hence, VHO algorithms are generally based on a predefined policy [3] that may consider different parameters including network condition, power consumption, network available bandwidth, security, cost, and other similar parameters.

Typically, VHO decision algorithms [4] should achieve seamless handoffs by adapting the decision to the application requirements, user preferences, and operating conditions. This adaptation requires cross-layer information exchange between different layers in the network protocol stack. Such information exchange becomes more important for real-time applications such as multi-media streaming that feature high diversity in the characteristics of streamed media and operating scenarios. In [5], the authors propose a generic link layer that sits below mobile IP in the protocol stack and enables information gathering form different network interfaces. The authors show that a significant performance improvement in packet loss can be realized by tuning the policy based decision using link layer information. More recently, the media Independent handover Functions (MIHF) standard, also known as IEEE 802.21, evolves as a generic framework for message exchange across different layers to facilitate VHO in heterogeneous wireless networks.

MIHF enables information exchange of several parameters, such as network condition, service cost, and application quality-of-service (QoS) requirements, in order to optimize VHO performance and facilitate VHO decision. IEEE 802.21 has received significant attention in different standardization groups. For example, IEEE 802.11 and IEEE 802.16 have respectively introduced the IEEE $802.11 \mathrm{u}$ and IEEE $802.16 \mathrm{~g}$ as amendments to their original standards to enable IEEE 802.21 functions [6]. Additionally, the IETF mobile IPv6 performance, signaling, and handoff optimization (MIPSHOP) is investigating mechanisms to support the reliable transmission of media independent handover messages. These standardization processes will speed the introduction of MIHF-enabled devices to the market and will enhance the performance of VHO algorithms.

The adaptive lifetime vertical handoff (ALIVE-HO) algorithm [7] is previously proposed as a highly adaptive VHO decision algorithm that considers different decision criteria including the network condition, handoff latency time ( $\mathrm{THO}_{\mathrm{HO}}$ and application QoS requirements. However, the study is based on theoretical analysis of physical and link layer parameters. Hence, it overlooks important practical operational aspects of higher layers. In this paper, we present a generic architecture for the mobile terminal (MT) stack incorporating MIHF and a connection coordinator for multi-interface devices. Additionally, we present the operation of ALIVE-HO using MIHF messages. Further more, we experimentally investigate the performance of ALIVE-HO and optimize its application signal strength threshold (ASST) for video streaming applications under different operating scenarios including various streaming rates and protocol stacks. 
The rest of this paper is organized as follows. Section II provides some background for VHO algorithms in wireless heterogeneous networks and an overview of the IEEE 802.21 standard. Section III explains the work flow and principles behind the ALIVE-HO algorithm and presents implementation guidelines for ALIVE-HO using the IEEE 802.21 standard. Section IV] presents the network testbed and the implementation specifics. Additionally, it presents our approach for determining ASST values for video streaming. Section V provides our conclusions and future work.

\section{BACKGROUND AND RELATED WORK}

The design of VHO decision is one of the main challenges in heterogeneous wireless networks due to the huge diversity in the characteristics of networks and services. This heterogeneity propels the concept of a preferred network, which is usually the network that provides better service at a lower cost. Hence, VHO decision algorithms define a set of rules that enable determining the best network in the user vicinity. In [3], the VHO decision is based on a periodic comparison of total network costs that are estimated as weighted sums of a normalized set of the VHO policy parameters. These weights vary according to user preferences and the MT status (e.g., power reserve).

Other works propose similar design approaches for $\mathrm{VHO}$ decision. For example, Zhu and McNair [8] introduce two cost-based policies including a collective handoff policy that estimates one cost for all the services and a prioritized multinetwork handoff policy estimates the cost for each service independently. Similarly, Chen et. al. [9] employ a handoff control center module to collect the information required for the VHO decision by monitoring the available MT interfaces and the system resources. This decision is also based on a score function that considers the usage expenses, link capacity, and power consumption for the available access technologies; and the MT uses the network that achieves the largest score. Similar approaches are presented in [10], [7], [11], [12] and the references therein. One main difficulty of the cost approach is its dependence on some parameters that are difficult to estimate, especially in large cellular networks, such as the available bandwidth, the channel condition, and the network user density, all of which change dynamically.

Alternatively, other VHO policies are based on a smaller set of parameters for practicality and ease of implementation. Assuming wireless local area network (WLAN) is the preferred technology to cellular network, Ylianttila et al. [13] present an algorithm to compute an optimization policy for a dwell timer according to the available data rates in both networks. In [14], Park et al. propose using a similar dwell timer-typed approach for both moving out of the preferred network (MO) and moving into the preferred network (MI) by performing the VHO if a specific number of the received beacons exceed or go below a predefined MI or MO threshold respectively. In [15], the authors employ a dwell timer during MO and use fast Fourier transform function to estimate the signal decay rate and compare it with a predefined threshold to take the

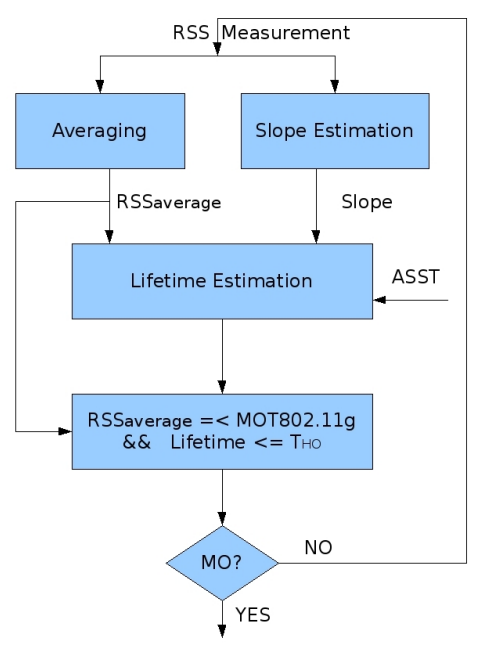

Figure 1. ALIVE-HO operation

MO decision. Additionally, the authors use a medium access layer sensing scheme to avoid MI to congested WLANs.

Similarly, ALIVE-HO [7] assumes a static preference for access technology with higher bandwidth. For example, always use an uncongested cheaper network whenever available is a basic rule for ALIVE-HO. The network congestion is induced through monitoring the network allocation vector in WLANs [15]. Another basic rule is to persist on using the preferred network as long as it satisfies the QoS requirements of the application and seamless handoff. The latter rule is realized by estimating the life-time of the mobile terminal within the preferred network. In this context, life-time corresponds to the duration over which the current access technology remains beneficial to the active applications and is expressed as

$$
E L[k]=\frac{\overline{R S S}[k]-\gamma}{S[k]} \leq T_{H O}
$$

where $k$ is the time step, $\overline{R S S}[k]$ represents an averaged RSS, $\gamma$ represents an application signal strength threshold, whose value is chosen to satisfy the requirements of the active applications, and $S[k]$ represents the RSS decay rate.

Figure 1 shows the operation of the ALIVE-HO algorithm on exiting the preferred network. Based on the measured and estimated parameters, the MT will proactively start the VHO procedure at time $k$ if the averaged received signal strength is less then or equal to a predefined exit threshold and the estimated lifetime is less than or equal to the handoff delay threshold, $T_{H O}$. The first condition indicates approaching the end of the current PoA coverage while the lifetime part tunes the handoff instant according to users mobility and application requirements to maximize the benefit from the preferred network characteristics.

MIHF [16] represents a framework for information exchange either locally between different layers or remotely between any two MIH-enabled entities to facilitate VHO decisions. Figure 2 shows the architecture of an MIH enabled device. Typically, MIHF will reside at 2.5 layer and interacts 


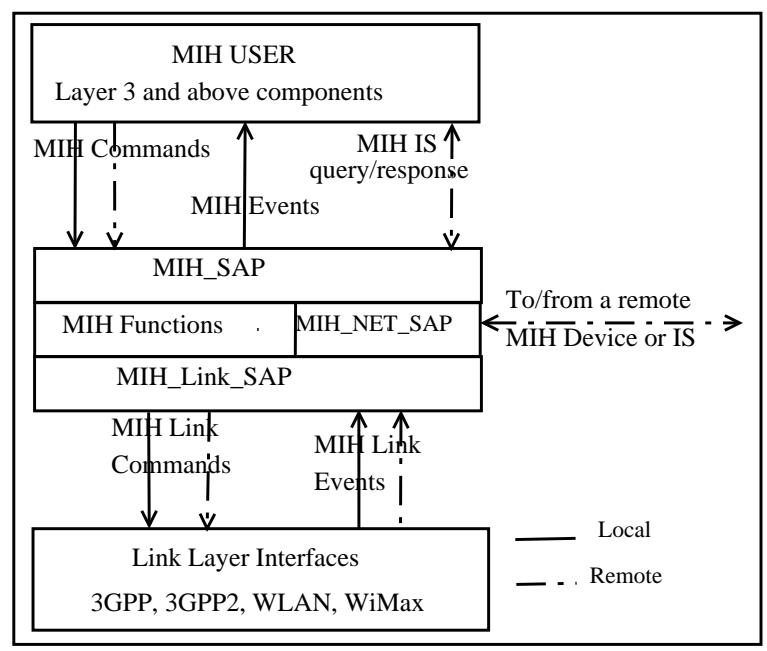

Figure 2. MIH Stack

with MIH users in upper layers and network interfaces in lower layers through the the MIH service access point (MIH_SAP) interface and the MIH_LINK_SAP respectively to enable cross layer information exchange. Additionally, MIH_NET_SAP is used to interact with remote MIH-enabled entities. Through these SAPs, MIH provides three different services including

- Information service by which an MIH-enabled entity can obtain information useful in device management and VHO decision.

- Command service by which higher layers can gather information from lower layers either locally or remotely and synchronously or asynchronously.

- Event service by which important information about lower layer performance are communicated to higher layers.

These services enables the mobile terminal to improve the VHO performance during all its phases including discovery, decision, and execution. For example, the event service enables the mobile device to perform proactive VHOs based on the provided information about network condition. Additionally, information service enables the device to save energy by only scanning the provided operating bands of neighbor PoAs. Several papers investigate the usage of IEEE 802.21 for VHO between different systems such as WiMax and WLAN [6] and $3 \mathrm{G}$ cellular and WiMax [17]. In [18], the authors propose a network initiated handoff based on IEEE 802.21.

Lampropoulos et al [19] presents how the emerging IEEE 802.21 standard enables seamless inter-technology handoff. In [20], Cacace et al show that the network performance experienced by the proposed a centralized connectivity manager interfacing with the 802.21 sublayer and responsible for the application of connectivity policies. Wan-Seon et al [21] partially implement the IEEE 802.21 framework to support seamless vertical handovers and efficient 802.11 AP discovery, and evaluate its performance in integrated IEEE 802.11/802.16e network. In [22], Mussabbir et. al. propose a framework for integrating IEEE 802.21 and fast mobile IP handover protocol. Additionally, they propose new information

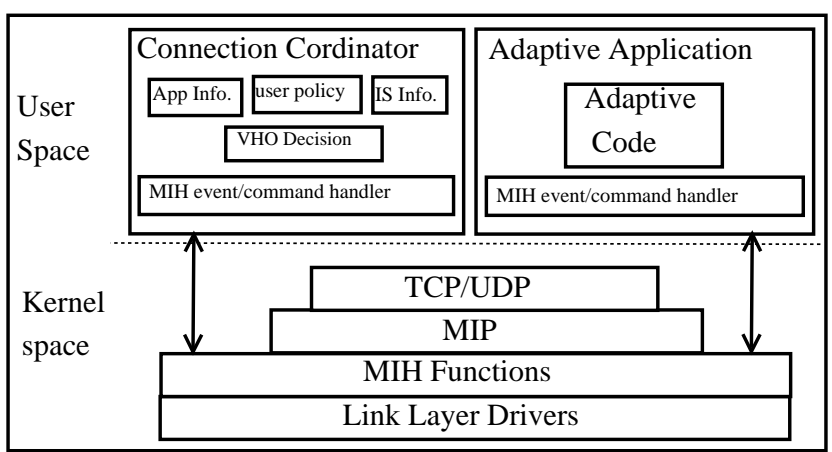

Figure 3. Mobile client architecture

element structure for exchanging application layer information across different layers through IEEE 802.21 framework. Such information would be useful for policy-based handoff decision algorithms.

These works only adopt simple hysteresis based VHO decision algorithms. In this work, we partially implement MIHF and integrate within ALIVE-HO algorithm to tune ALIVEHO for optimal video streaming performance in integrated networks.

\section{iII. Mobile Client Operation}

Figure 3 shows the protocol stack for a multi-interface mobile client. In the shown architecture, 802.21 enables important functions for the performance of proactive VHO. Additionally, the Connection Coordinator (CC) represents the heart of VHO management by participating in different functions including mobility management, power management, and resource management.

\section{A. Downward VHOs}

Typically, ALIVE-HO prefers using the cheaper networks whenever they have sufficient resources. Hence, on using an expensive network, such as cellular networks, the CC actively scans for cheaper networks. However, to save the power used in this scanning, the CC proactively consults the MIH information server for information about the neighbor PoAs including operating bands, communication cost, and Medium Access Control addresses. On discovering a new PoA, the MIH informs the CC using Link_Up message. If the discovered network is cheaper than the current one, the CC checks for resource availability in this network using MIHF resource query-response procedure. If the $\mathrm{CC}$ receives a positive response from the candidate PoA, it starts the VHO procedure including network authentication, address configuration, and traffic redirection.

\section{B. Upward VHOs}

Generally, upward VHOs usually correspond to moving from a cheaper resourceful network to a more expensive and resource-limited network. For example, moving from a WLAN to a cellular network. Hence, users will persist on using this preferred network as long as it satisfies the QoS requirements 
of active applications. Consequently, on using a preferred network, the CC registers for two different MIHF events to take the VHO decision using ALIVE-HO.

1) MIH_Link_Parameter_Report event that indicates that the drop of average RSS below the target exit threshold.

2) MIH_Link_Going_Down event indicates an expected loss of the connection to the current point of attachment within a specific duration, e.g. connection will be lost in two seconds.

In order to register for these events, the $\mathrm{CC}$ needs to instruct the MIHF with the thresholds associated with such events. These thresholds are communicated using the MIH_Link_Configure_Thresholds command through the MIH_SAP. Generally, the preferred network exit threshold $(E T)$ is a few $\mathrm{dB}$ (5-7) above the interface sensitivity $\left(I_{s}\right)$ while the ASST value used in triggering the MIH_Link_Going_Down message is obtained from a database of thresholds that correspond to the the active application QoS requirement. Using ALIVE-HO as a VHO algorithm, the CC will configure MIH_Link_Going_Down duration to the expected handoff delay $\left(T_{H O}\right)$ required for completing the proactive signaling for resource query, authentication, and address configuration with the new PoA.

\section{EXPERIMENTATION AND ASST OPTIMIZATION}

In this section, we first present a description for our testbed and implementation specifics of some MIHF functions. We then present our experimentation procedure and the performance metrics used to determine the most effective ASST.

\section{A. Testbed Setup and MIHF Implementation}

In our experiments, we assume the mobile terminal is initially located in its preferred network. The testbed consists of a Dell Latitude D610 running Ubuntu as mobile client, a WLAN access point connected to an Ubuntu based router that is also connected to a Darwin streaming server installed in another machine. The mobile client is equipped with a Netgear WPN511 RangeMax wireless interface and uses videoLan [23] as a streaming client application. The algorithm and $\mathrm{MIH}$ functions are implemented in the $\mathrm{C}$ programming language in user space. The link layer information RSS value from the currently connected AP is achieved by using the Linux system call ioctl, which allows user communication between user space and kernel space.

On implementing the MIHF link parameter change and link going down, we use simple a moving average estimators. At any time sampling instant $k$, the average RSS, denoted as $\overline{R S S}[k]$ is expressed as

$$
\overline{R S S}[k]=\frac{1}{W a v} \sum_{i=0}^{W a v-1} R S S[k-i],
$$

where $W$ av represents the averaging window size. Similarly, the RSS rate of change, denoted as $S[k]$, is estimated using moving average window and is expressed as
Table I

PARAMETER VALUES

\begin{tabular}{|c|c|c|c|c|c|c|c|}
\hline Param. & $I_{s}$ & $E T$ & $V$ & $T_{s}$ & $D_{a v}$ & $D_{s}$ & $T_{H O}$ \\
\hline Value & $-90 \mathrm{dBm}$ & $-85 \mathrm{dBm}$ & $2 \mathrm{~m} / \mathrm{s}$ & $0.1 \mathrm{~s}$ & $0.5 \mathrm{~m}$ & $5 \mathrm{~m}$ & $1 \mathrm{~s}$ \\
\hline
\end{tabular}

$$
S[k]=\frac{M_{1}[k]-M_{2}[K]}{W_{S} T_{S}},
$$

where $W_{s}$ and $T_{s}$ represent the slope estimator window size and the sampling interval respectively, $M_{1}[k]$ and $M_{2}[k]$ correspond to the average of $\overline{R S S}[k]$ during the first half and second half of the slope estimator window and are expressed as

$$
\begin{aligned}
& M_{1}[k]=\frac{2}{W_{s}} \sum_{i=0}^{\frac{W s}{2}-1} \overline{R S S}[k-W s+1+i] \\
& M_{2}[k]=\frac{2}{W_{s}} \sum_{i=\frac{W s}{2}}^{W s-1} \overline{R S S}[k-W s+1+i]
\end{aligned}
$$

The window sizes used for estimating the RSS average and decay rate are estimated as

$$
\begin{gathered}
W_{a v}=\max \left(10,\left\lfloor\frac{D a v}{V T s}\right\rfloor\right) \\
W_{s}=2 * \max \left(50,\left\lfloor\frac{D s}{V T s}\right\rfloor\right)
\end{gathered}
$$

where $D_{a v}$ and $D_{s}$ represent window distance in meters and $V$ represents the user moving speed, which can be obtained using one of the available velocity estimators. The Link_Going_Down interval is estimated using the same expression for estimated lifetime in ALIVE-HO, i.e. $E L[k]=$ $\frac{\overline{R S S}[k]-\gamma}{S[k]}$.

\section{B. Experimental Procedure and Performance Metrics}

In our experiments, the mobile device is carried away from the access point at a walking speed while the media is streamed from the server. The values of important parameters in our experiments are shown in Table $\mathrm{I}$. Note that $T_{H O}$ generally depends on the integrated network architecture, specifically the time required for authentication and address configuration. In our performance evaluation, we estimate the performance metrics over a handoff transition duration, which we consider the four seconds preceding exiting the preferred network, i.e. the four second exactly before completing the VHO. This enables us to properly asses the impact of the handoff decision timing on the streaming performance and avoid misleading results that are influenced by using the wireless network in a good condition away from the PoA coverage edge.

We use wireshark to observe packets passing through the client interface and facilitate determining the frames transmitted during the handoff transition area. The set of frames transmitted over the four seconds handoff transition period is denoted as Videorecord.cut. We also obtain the corresponding set of frames from the original video, denoted as Videooriginal.cut. Using these video fragments, we use the MSU Video Quality Measurement Tool [24] to evaluate the 


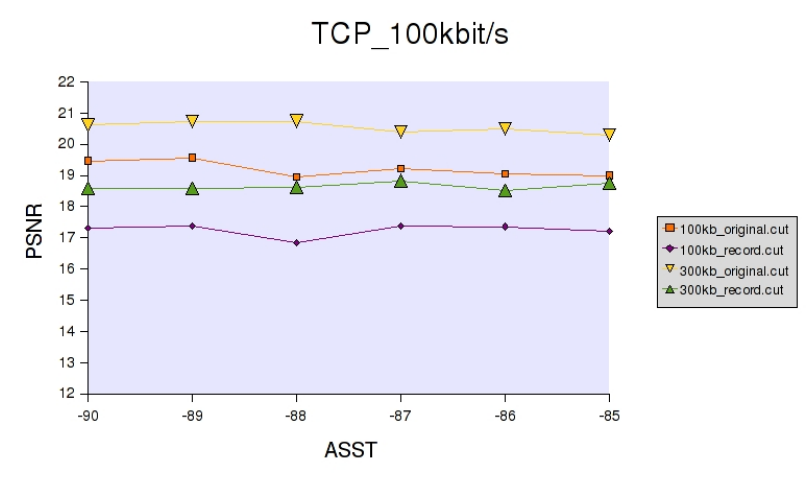

Figure 4. PSNR comparison based on RTSP/TCP

Table II

FRAMES LOST CORRESPONDING TO ASST

\begin{tabular}{|c|c|c|c|c|c|c|}
\hline ASST & -90 & -89 & -88 & -87 & -86 & -85 \\
\hline Lost frame_100kbit/s & 13.3 & 5 & 3 & 0.67 & 0 & 0 \\
\hline Lost frame_300kbit/s & 38 & 28 & 4 & 0.33 & 0 & 0 \\
\hline
\end{tabular}

streaming quality by estimating the peak signal to noise ratio (PSNR) and number of lost frames. The PSNR is most commonly used as a measure for quality of reconstruction of lose compression codecs (e.g. for image compression). In our case, the image packets lost due to bad network conditions will obviously increase the noise and in turn reduce the PSNR of the video. To satisfy users QoS, we needed to compare the average PSNR between streamed media to the MT and the original stream to determine the most appropriate practical value of ASST in the ALIVE-HO algorithm.

\section{Determination of ASST Values}

Figure 4 shows the PSNR for RTSP/TCP stream versus ASST using $100 \mathrm{kbit} / \mathrm{s}$ and $300 \mathrm{kbit} / \mathrm{s}$ videos. The figure shows a small drop between the PSNR of the original and streamed media that is usually related to encoding and decoding processes [25]. Additionally, the figure shows limited impact for ASST value on the PSNR because TCP as a reliable network protocol persists on retransmitting any lost or corrupted packets. However, for ASST less than $-87 \mathrm{dBm}$, a large number of packet retransmission take place during the handoff transition duration. These retransmissions affect the video playout quality and lead to playout interruption.

Figure 5 shows the PSNR for RTP/UDP stream versus ASST using $100 \mathrm{kbit} / \mathrm{s}$ and $300 \mathrm{kbit} / \mathrm{s}$ videos. In contrast to TCP, UDP does not transmit any lost or corrupted packets. This clearly leads to a noticeable drop in PSNR as ASST decreases below $-87 \mathrm{dBm}$. Tables $\Pi$ show the lost frames during the handoff transition area for the received stream. The lost frames may lead to significant deterioration for the perceived image quality as shown in Figure 6. Based on the obtained results and our subjective testing, the optimal value for UDP based streaming is chosen as $-86 \mathrm{~dB}$.

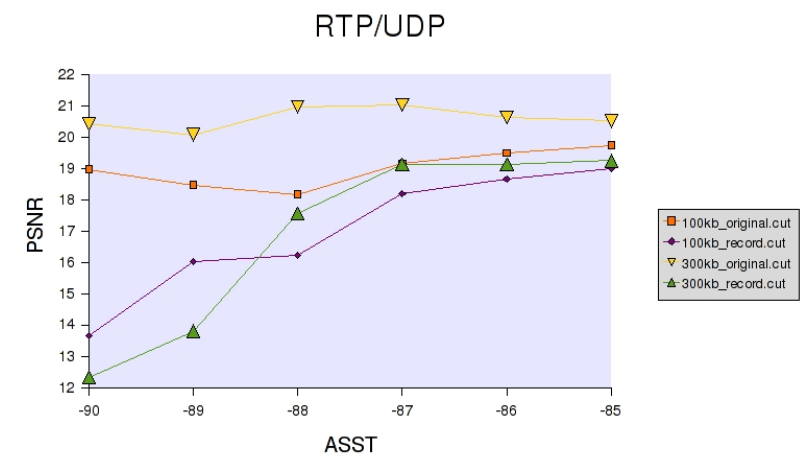

Figure 5. PSNR comparison based on RTP/UDP

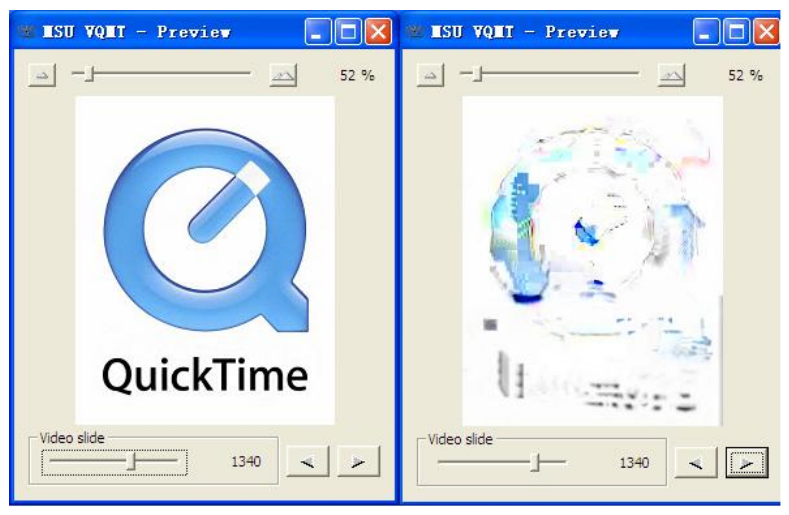

Figure 6. Subjective frame comparison

To this end, it is worth noting that the obtained values corresponds are specific to the investigated scenario. Similar experiments are to be conducted for different other parameters such as streaming rates and protocols to build a suitable database containing the optimal ASST values under different scenarios. Since the development of this database is not expected to be exhaustive, interpolation techniques can be used to get an approximate values for missing entries in the database.

\section{CONCLUSions}

The integration of different access technologies introduces VHOs as a unique challenge. The success of such integrated systems is conditioned on achieving seamless VHOs that are transparent to the user. To achieve this, information exchange across different layers should be enabled and new application adaptive frameworks should be developed. In this paper, we presented a generic mobile terminal architecture based on the state of the art standard IEEE 802.21 to facilitate seamless VHO for streaming applications. Additionally, we optimize the performance of the chosen VHO algorithm to ensure seamless streaming under different operating scenarios. As a future work, we plan to test the impact of window shape on the performance of VHO. Additionally, we are also interested in 
investigating the adaptation of video streaming rate according to the network condition.

\section{ACKNOWLEDGMENT}

The authors wish to acknowledge the support of Science Foundation Ireland (SFI).

\section{REFERENCES}

[1] M. Buddhikot, G. Chandranmenon, S. Han, W. L. Y., S. Miller, and L. Salgarelli, "Integration of 802.11 and Third-Generation Wireless Data Networks," INFOCOM 2003. Twenty-Second Annual Joint Conference of the IEEE Computer and Communications. IEEE Societiess, vol. 1, pp. 503-512, April 2003.

[2] G. P. Pollini, "Trends in handover design," IEEE Commun Mag, vol. 32, no. 3, pp. 82-90, March 1996.

[3] H. Wang, R. H. Katz, and J. Giese, "Policy-Enabled Handoffs across Heterogeneous Wireless Networks," in Proc. of the Second IEEE Workshop on Mobile Computer Systems and Applications, New Orleans, Louisiana, Feb. 1999, p. 51.

[4] M. Kassar, B. Kervella, and G. Pujolle, "An overview of vertical handover decision strategies in heterogeneous wireless networks," Computer Communications, vol. 31, no. 10, pp. 2607-2620, June 2008.

[5] Stefan Aust and Daniel Proetel and Nikolaus A. Fikouras and Carmelita Görg and Cornel Pampu, "Policy based Mobile IP handoff decision (POLIMAND) using generic link layer information," in in Proc. 5th IEEE Int. Conf. Mobile and Wireless Commun. Netw, 2003.

[6] A. Pontes, D. dos Passos Silva, J. Jailton, O. Rodrigues, and K. L. Dias, "Handover management in integrated WLAN and mobile WiMAX networks," IEEE Wireless Commun., vol. 15, no. 5, pp. 86-95, Oct. 2008.

[7] A. H. Zahran, B. Liang, and A. Saleh, "Signal threshold adaptation for vertical handoff in heterogeneous wireless networks," Mob. Netw. Appl., vol. 11 , no. 4, pp. 625-640, August 2006.

[8] F. Zhu and J. McNair, "Optimizations for vertical handoff decision algorithms," in Proc. of IEEE Wireless Commun. and Networking Conference (WCNC'04), vol. 2, March 2004, pp. 867 - 872.

[9] L. Chen, T. Sun, B. Chen, V. Rajendran, and M. Gerla, "A Smart Decision Model for Vertical Handoff," in Proc. of the 4th Int'l Workshop on Wireless Internet and Reconfigurability (ANWIRE'04), May 2004.

[10] J. McNair and F. Zhu, "Vertical Handoffs in Fourth Generation (4G) Multi-network Environments," IEEE Wireless Commun. Mag., vol. 11, no. 3 , pp. $8-15$, June 2004

[11] E. Stevens-Navarro and V. Wong, "Comparison between Vertical Handoff Decision Algorithms for Heterogeneous Wireless Networks," in Proc. of IEEE 63rd Vehicular Technology Conference, 2006 (VTC 2006Spring), vol. 2, 2006, pp. 947-951.

[12] M. Kassar, B. Kervellaa, and G. Pujolle, "An overview of vertical handover decision strategies in heterogeneous wireless networks," Computer Communications, vol. 31, no. 10, pp. 2607-2620, 2008.

[13] M. Ylianttila, M. Pande, J. Makela, and P. Mahonen, "Optimization scheme for mobile users performing vertical hand-offs between IEEE 802.11 and GPRS/EDGE networks," in Proc. of IEEE Global Telecommunications Conference GLOBECOM'01, vol. 6, San Antonio, Texas, USA, Nov. 2001, pp. 3439 - 3443

[14] H. Park, S. Yoon, T. Kim, J. Park, M. Do, , and J. Lee, "Vertical handoff procedure and algorithm between ieee802.11 WLAN and CDMA cellular network," in Proc. CDMA Int'l Conf., 2002, pp. 103-112.

[15] Q. Zhang, C. Guo, Z. Guo, and W. Zhu, "Efficient mobility management for vertical handoff between WWAN and WLAN," IEEE Commun. Mag., vol. 41, no. 11, pp. 102 - 108, Nov. 2003.

[16] IEEE, "Local and Metropolitan Area Networks: Media Independent Handover Services," IEEE P802.21/D11.0 Draft Standard, May 2008.

[17] J.-H. Seol and J.-M. Chung, "IEEE 802.21 MIH based Handover for Next Generation Mobile Communication Systems," in Proc. of 4th International Conference onInnovations in Information Technology (Innovations '07), 2007, pp. 431-435. [Online]. Available: http: //dx.doi.org/10.1109/IIT.2007.4430458

[18] J.-Y. Baek, D.-J. Kim, Y.-J. Suh, E.-S. Hwang, and Y.-D. Chung, "Network-Initiated Handover Based on IEEE 802.21 Framework for QoS Service Continuity in UMTS/802.16e Networks," in Proc. IEEE Vehicular Technology Conference VTC Spring 2008, May 11-14, 2008, pp. 2157-2161.
[19] G. Lampropoulos, A. Salkintzis, and N. Passas, "Media-independent handover for seamless service provision in heterogeneous networks," IEEE Commun Mag, vol. 46, no. 1, pp. 64-71, January 2008.

[20] F. Cacace and L. Vollero, "Managing mobility and adaptation in upcoming 802.21 enabled devices," in Proc of the 4th international workshop on Wireless mobile applications and services on WLAN hotspots (WMASH '06). New York, NY, USA: ACM, 2006, pp. 110 .

[21] W.-S. Lim, D.-W. Kim, Y.-J. Suh, and J.-J. Won, "Implementation and performance study of IEEE 802.21 in integrated IEEE 802.11/802.16e networks," Comput. Commun., vol. 32, no. 1, pp. 134-143, 2009.

[22] Q. Mussabbir, W. Yao, Z. Niu, and X. Fu, "Optimized FMIPv6 Using IEEE 802.21 MIH Services in Vehicular Networks," IEEE Trans. on Veh. Tech., vol. 56, no. 6, pp. 3397-3407, Nov. 2007.

[23] "VideoLAN - VLC media player." [Online]. Available: http://www. videolan.org/

[24] "MSU Video Quality Measurement Tool." [Online]. Available: http://compression.ru/video/quality_measure/video_measurement_ tool_en.html

[25] J. M. F. Moura, R. S. Jasinschi, H. Shiojiri, and J. C. Lin, "Retrieving quality video across heterogeneous networks. Video over wireless," IEEE Personal Commun., vol. 3, no. 1, pp. 44-54, February 1996.

[26] P. Chan, R. Sheriff, Y. Hu, P. Conforto, and C. Tocci, "Mobility management incorporating fuzzy logic for heterogeneous a IP environment," Communications Magazine, IEEE, vol. 39, no. 12, pp. 42-51, December 2001.

[27] P. Goyal and S. K. Saxena, "A Dynamic Decision Model for Vertical Handoffs across Heterogeneous Wireless Networks," World Academy of Science, Engineering and Technology, vol. 31, pp. 677-682, July 2008.

[28] V. G. Gupta and D. Johnston, "IEEE 802.21 A Generalized Model for Link Layer Triggers," March 1st 2004. [Online]. Available: http://www.ieee802.org/handoff/march04_meeting_doc

[29] A. Hasswa, N. Nasser, and H. Hassanein, "Tramcar: A Context-Aware Cross-Layer Architecture for Next Generation Heterogeneous Wireless Networks," in Proc. of IEEE International Conference on Communications (ICC'06), vol. 1, June 2006, pp. 240-245.

[30] Y. G. KIM, T. S. Park, J. H. Kwon, D. Y. Joung, J. S. Park, S. K. Kim, C. H. Lee, and J. W. Song, "System and method for real-time video quality assessment based on transmission properties," Patent. [Online]. Available: http://www.faqs.org/patents/app/20090153668

[31] J. Lazzaro, "Framing Real-time Transport Protocol (RTP) and RTP Control Protocol (RTCP) Packets over Connection-Oriented Transport," Network Working Group of the Internet Engineering Task Force (IETF), July 2006. [Online]. Available: http://www.rfc-editor.org/rfc/rfc4571.txt

[32] J. Makela, M. Ylianttila, and K. Pahlavan, "Handoff decision in multiservice networks," in Proc. of the 11th IEEE International Symposium on Personal, Indoor and Mobile Radio Communications (PIMRC 2000), vol. 1, Sep. 2000, pp. 655-659.

[33] H. Schulzrinne, U. Columbia, A. Rao, and R. Lanphier, "Real Time Streaming Protocol," RFC 2326, April 1998. [Online]. Available: http://www.ietf.org/rfc/rfc2326.txt

[34] A. H. Zahran and C. J. Sreenan, "Pseudo-Optimal Greedy Media Streaming Algorithm for Heterogeneous Wireless Networks," in Proc. of IEEE International Conference on Wireless and Mobile Computing Networking and Communications (WIMOB '08), Oct. 2008, pp. 390396. 